\title{
Unusual presentation and inconclusive biopsy render fibroadenoma in two young females a diagnostic dilemma
}

Debra Meerkotter, MB BCh, FCRad (D) SA

Regional Imaging, St Vincents Hospital, Launceston, Tasmania, Australia

Savvas Andronikou, MB BCh, FCRad, FRCR, $P h D$

Department of Radiology, University of Cape Town

\section{Abstract}

Two young, nonlactating, nulliparous women presented with acutely painful breast masses. Sonographic features showed mixed echogenic masses. Core biopsies were not diagnostic, and surgical excision revealed infarcted fibroadenomas in both cases. Although fibroadenomas are common, they do not commonly infarct, and only rarely in non-lactating or non-pregnant females. These two cases highlight the clinical and imaging characteristics of an important differential diagnosis.

\section{Introduction}

\section{Case 1}

A 15-year-old girl had been aware of a left breast lump for several months. She presented acutely with severe pain in the mass. On examination, the left breast was erythematous, slightly warm and tender, particularly over the mass. The mass itself was firm but mobile. High-frequency linear ultrasound of the left breast demonstrated a solitary mass which was predominantly solid with an ill-defined area of decreased echogenicity within it and a linear area of anechogenicity within the latter. There was a capsule around the anechogenic area which was echogenic. No posterior acoustic shadowing was present, and it measured at least $30 \times 40 \times 45 \mathrm{~mm}$ in size (Figs 1a and $1 \mathrm{~b}$ ). A reactive lymph node in the left axilla was noted. The right breast and axilla were within normal limits. A core biopsy of the mass revealed benign features with areas of extensive infarctoid necrosis, abscess formation and a cellular, necrotic proliferation. Complete local excision was recommended due to the extensive necrosis, and a $50 \times 40 \times 30 \mathrm{~mm}$ mass weighing 34 grams was excised. Histological examination showed the presence of an infarcted juvenile (cellular) fibroadenoma with extensive areas of

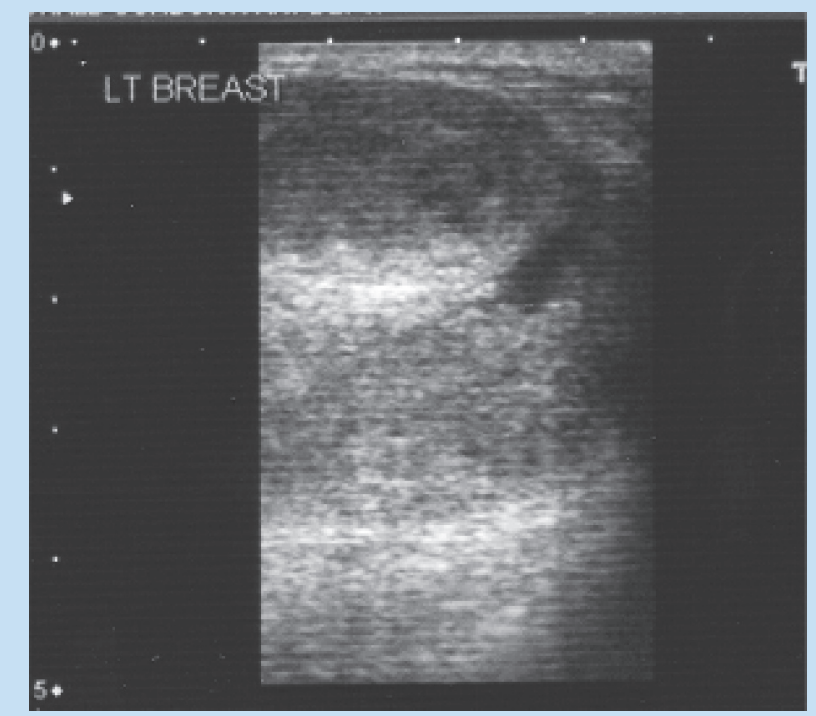

Fig. 1a. Case 1: High-frequency linear ultrasound left breast masstransverse section.

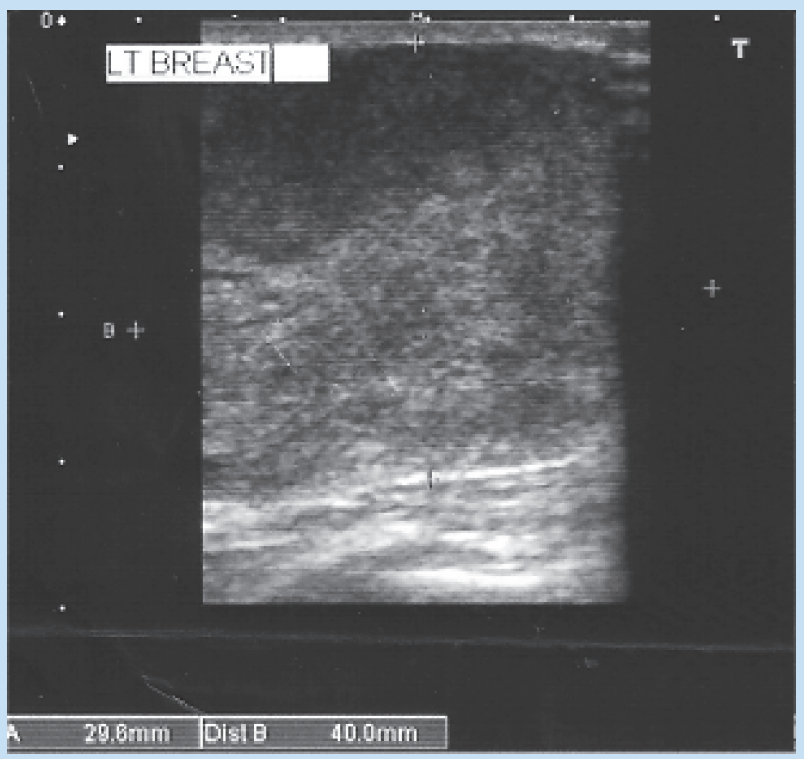

Fig. 1b. Case 1: High-frequency linear ultrasound of left breast mass longitudinal section. 
infarctoid necrosis. Viable areas showed no evidence of atypia or malignancy. Areas of suppurative inflammation were also noted, suggesting superinfection within the infarcted areas. The diagnosis of an infarcted fibroadenoma was made.

\section{Case 2}

A 19-year-old woman presented with a lump in the right breast which had recently become painful. She was not pregnant or lactating. On examination, a palpable mass was noted in the right breast

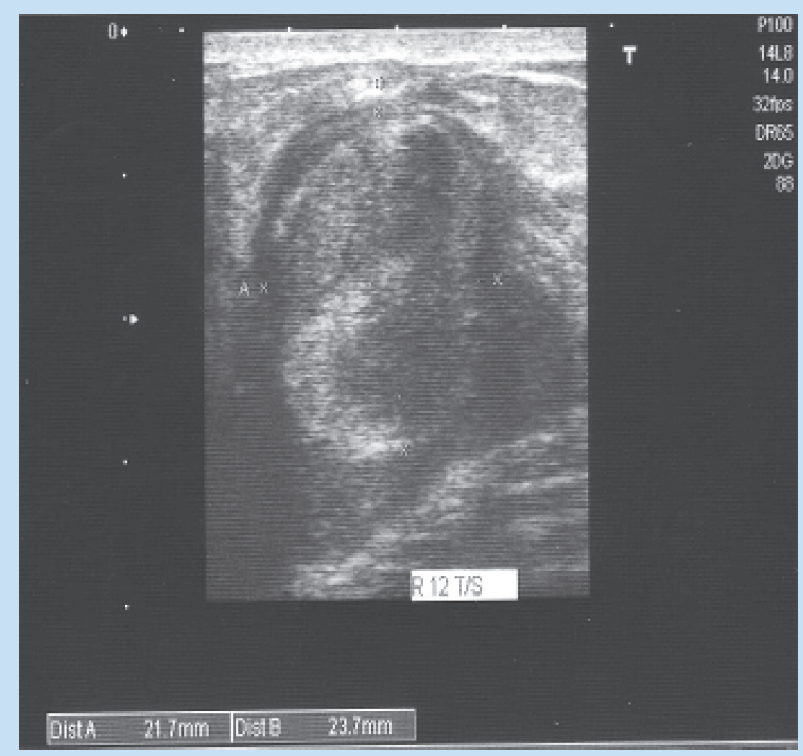

Fig. 2a. Case 2: High-frequency linear ultrasound of lesion in right breast - transverse section.

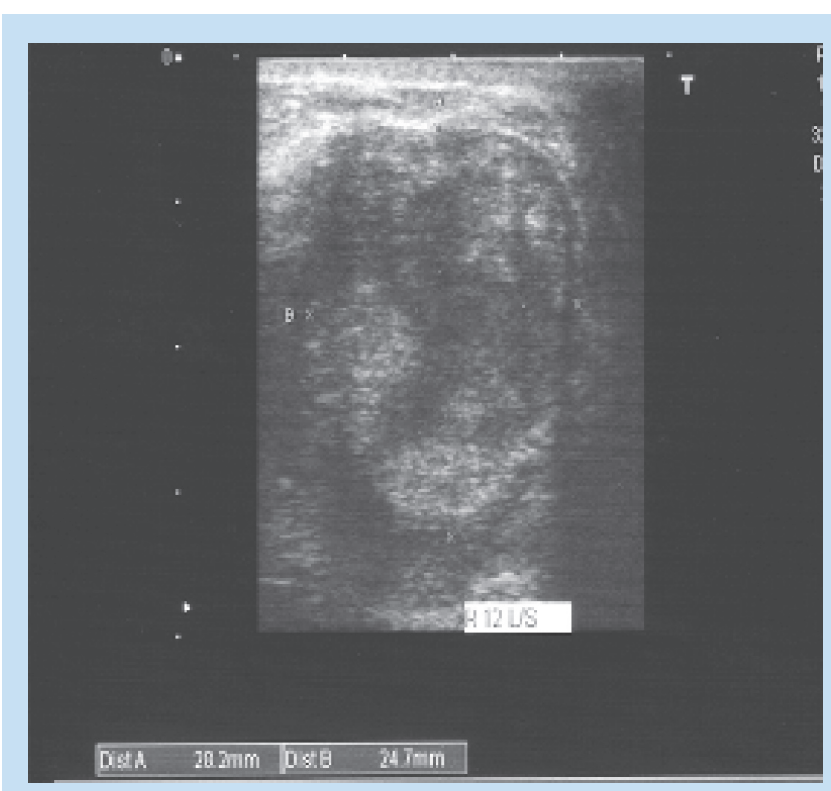

Fig. 2b. Case 2: High-frequency linear ultrasound of lesion in right breast - longitudinal section. in the 12 o clock position. The overlying skin was not inflamed. On ultrasound examination, there was a rounded space-occupying lesion measuring $22 \times 24 \times 28 \mathrm{~mm}$ in size, just above the areola. It was of mixed echogenicity, and the superficial border was well defined by an echogenic capsule. The deep border was ill defined (Figs $2 \mathrm{a}$ and $2 \mathrm{~b}$ ). The mass was solitary. A core biopsy revealed morphological features suggestive of an inflammatory lesion consistent with a resolving mastitis. A wide local excision of the lesion was performed and breast tissue measuring $40 \times 25 \times 25 \mathrm{~mm}$ in was excised. A poorly circumscribed lesion measuring $30 \times 25 \mathrm{~mm}$ and weighing $21.5 \mathrm{~g}$ was found. Histological analysis showed an infarcted benign fibroadenoma. The tumour was well circumscribed with a lobulated appearance. Dense fibrous tissue was present with a large area of coagulative necrosis with areas of haemorrhage. No atypia or malignancy was noted. The lesion was completely excised.

\section{Discussion}

The presence of fibroadenomas in female adolescents is well documented; these are generally observed over time without complications. Fibroadenomas are known to infarct in approximately $0.5-1.5$ $\%$ of cases. The rare case of spontaneous infarction generally occurs in three known settings. Performing a fine-needle aspiration (FNA) is a known predisposing factor in precipitating infarction. ${ }^{1}$ This type of infarction is not common but has been documented following FNA of thyroid nodules, salivary glands, breast fibroadenomas, lymph nodes and renal cell carcinomas. The other predisposing factors are pregnancy and lactation. ${ }^{2}$ The increased metabolic demands of pregnancy and lactation are thought to result in a relative vascular ischaemia and infarction in these settings. ${ }^{3}$ A rare case of spontaneous infarction of a fibroadenoma in a postmenopausal woman has been documented. ${ }^{4}$

In our two patients, spontaneous infarction of a fibroadenoma had occurred. Neither patient was pregnant nor lactating, and no previous FNA had been performed. This process has previously been documented as a rare and unique occurrence ${ }^{3}$ in the absence of factors predisposing to infarction.

On ultrasound, the masses were predominantly solid but contained mixed echogenicity within them. They no longer appeared to have the classic features of a fibroadenoma. Sonographic distinction from a phyllodes tumour was not possible. In both patients, core biopsy results were not diagnostic of the condition, and full diagnosis was obtained only on surgical excision. This entity may be confused with mastitis, duct ectasia, tuberculosis of the breast, and carcinoma on cytology smears. ${ }^{5}$ A combination of clinical examination and ultrasound and histology findings are essential for the diagnosis, which can be aided by an awareness of this rare complication.

\section{Conclusion}

Although a rare complication, infarction of fibroadenomas should be considered in the acutely painful deterioration of a breast mass. 


\section{CASE REPORT}

The salient clinical features in our report were that both cases were young healthy females and that the masses had recently become painful. The sonographic features show nonspecific mixed echo patterns, which would reflect the process of infarction of a solid mass.

1. Pinto RG, Couto F, Mandreker S. Infarction after fine needle aspiration. A report of four cases. Acta Cytol 1996; 40(4): 739-74
2. Raju GC, Naraynsingh V. Infarction of fibroadenoma of the breast. J Royal Coll Surg Edinb 1985; 30 $162-163$.

3. Fowler CL. Spontaneous infarction of fibroadenoma in an adolescent girl. Pediatr Radiol 2004; 34: 988990.

4. Ichihara S, Matsuyama T, Kubo K, Tamura Z, Aoyama H. Infarction of breast fibroadenoma in a postmenopausal woman. Pathol Int 1994; 44(5): 398-400.

5. Kamayani M. Diagnostic difficulties in spontaneous infarction of a fibroadenoma in an adolescent: case report. Diag Cytopathol 2002; 26(1): 26-28

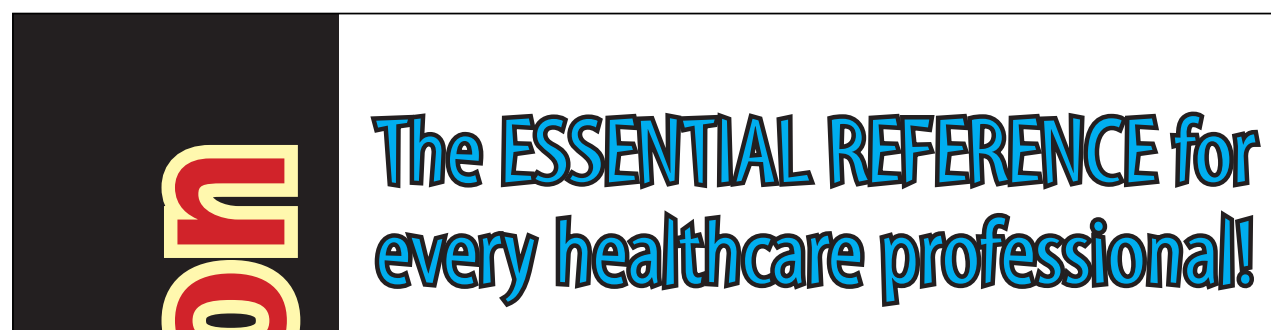

The carefully and thoroughly updated $9^{\text {th }}$ edition of the South African Medicines Formulary (SAMF) can now be ordered. It is your essential reference to rational, safe and cost-efficient use of medicines. That is why you should not prescribe without it.

The newly published SAMF provides easy access to the latest, most scientifically accurate information - including full drug profiles, clinical notes and special prescriber's points. The convenient pocket-size design enables you to fit it comfortably into your bag or hospital coat pocket - always at hand for ready reference.

\section{WHYYOU SHOULDNTT BE WITHOUT THE SAMF 9TH EDITION}

The new $9^{\text {th }}$ edition of SAMF provides expanded information on key issues facing South African healthcare professionals today, including antiretrovirals, TB treatment guidelines, management guidelines for asthma and chronic heart failure, other common chronic conditions and prescribing in sport.

- It presents practical, new approaches to the management of venomous bites and stings.

- It outlines extensively the acute adverse reactions to drugs of abuse, and their management.

- It features new as well as existing drugs, indexed by both trade and generic names.

- It offers fresh insights into informed prescribing and carries cautionary guidelines on drug interactions and a range of special risk patients and conditions.

And, as always, you can rely on.

- the professional compilation and editing by a team from the Division of Clinical Pharmacology, UCT

- an independent and unbiased guide on prescribing in South Africa today

- the indication of agents included in the SA and WHO essential drug lists

- support of the SA national drug policy

- guidance for prescribing during pregnancy and lactation, and in patients with porphyria, liver disease and renal impairment (including tables with drug dosage adjustments); and

- indexed and page tabs for quick and easy access to each section.

\section{YOUR SATISFACTION IS GUARANTEED}

\section{3 easy order options:}

1. PHONE EDWARD OR BYRON - 0216817000

2. FAX the completed SAMF order form to

0866006218

3. EMAIL: edwardm@hmpg.co.za OR byronm@hmpg.co.za

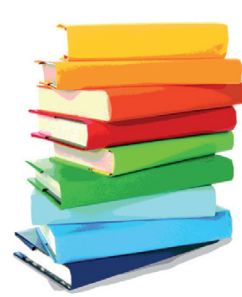

